\title{
CDISC SDTM West Haven Hepatic Encephalopathy Grade Terminology
}

National Cancer Institute

\section{Source}

National Cancer Institute. CDISC SDTM West Haven Hepatic Encephalopathy Grade

Terminology. NCl Thesaurus. Code C117746.

Terminology associated with the West Haven hepatic encephalopathy grade codelist of the Clinical Data Interchange Standards Consortium (CDISC) Study Data Tabulation Model (SDT M). 